\title{
The algae names and collection of the Spanish Phycologist Pedro González Guerrero
}

\author{
Jose Luis Izquierdo $^{1 *} \&$ Francisco Pando ${ }^{2}$ \\ ${ }^{1}$ Centro de Investigación, Seguimiento y Evaluación, Parque Nacional Sierra de Guadarrama, Comunidad de Madrid, \\ Spain; joseluis.izquierdo@pnsg.es \\ ${ }^{2}$ Real Jardín Botánico de Madrid, CSIC, Plaza de Murillo 2, 28014 Madrid, Spain
}

\begin{abstract}
Izquierdo, J.L. \& Pando, F. 2017. The algae names and collection of the Spanish Phycologist Pedro González Guerrero. Anales Jard. Bot. Madrid 74(1): e047.

In this paper we collect the 103 algae names published by the Spanish phycologist Pedro González Guerrero during his life-long study of algae at the Royal Botanic Garden of Madrid. Names are presented with specific information to facilitate the typification of these names in the future, since P. González only used small illustrations as type elements when describing new infrageneric taxa. We also review here the status of this collection, which has been restored and databased in the process. The collection currently comprises over 2150 specimens, a fraction of what it was a much larger one.
\end{abstract}

Keywords: Algae, Historical Collections, Nomenclature.

\begin{abstract}
Resumen
Izquierdo, J.L. \& Pando, F. 2017. Colección y nombres de algas del ficólogo español Pedro González Guerrero. Anales Jard. Bot. Madrid 74(1): e047.

En este trabajo reunimos los 103 nombres de alga que el ficólogo español Pedro González Guerrero publicó a lo largo de toda una vida científica dedicada al estudio de este grupo en el Real Jardín Botánico de Madrid. Revisamos el estado de su colección, la cual ha sido restaurada y registrada en una base de datos, y proporcionamos información detallada de los nombres y ejemplares para facilitar la tipificación en el futuro, puesto que P. González Guerrero se limitaba a usar pequeñas ilustraciones como elementos tipo al describir nuevos táxones infraespecíficos. La colección actual abarca más de 2150 muestras y es solo una fracción de la original. Palabras clave: Algas, colecciones históricas, nomenclatura.
\end{abstract}

ORCID ID: J.L. Izquierdo (http://orcid.org/0000-0002-0012-931X); F. Pando (http://orcid.org/0000-0002-6321-9398).

Received: 11-XI-2016; accepted: 20-I-2017; published online: 28-IV-2017; Associate Editor: Margarita Dueñas.

Copyright: ( 2017 CSIC. This is an open-access article distributed under the terms of the Creative Commons Attribution-Non Commercial (by-nc) Spain 3.0 License.

\section{INTRODUCTION}

Pedro González Guerrero (1902-1984) was a very active phycologist, and the first Spanish botanist who studied freshwater algae and estuaries. He collected systematically and extensively continental algae in the Iberian Peninsula, as well as in other countries -Morocco, Argentina, Norway, and Greece-. González Guerrero published 86 scientific works (Álvarez-Cobelas \& Gallardo, 1985), and proposed 103 new taxa: 5 genera, 74 species, 22 varieties, and 2 forms (González Guerrero, 1929-1965). He carried out most of his research at the Royal Botanic Garden of Madrid between 1923 and 1974, where he was head of the Sección de Ficología from 1952 to 1969 (Álvarez-Cobelas \& Gallardo, 1985). The algae collection contained in the herbarium of this institution - MA - largely reflects the investigations conducted by González Guerrero. His work is an essential reference in Iberian freshwater phycology.

The aim of this paper is to make known this collection as well as to provide the necessary information to asses it adequately and to facilitate future typification of P. González's names.

\section{MATERIAL AND METHODS}

Algae in González Guerrero's collection are preserved in liquid - formalin 4\%-, in glass test tubes. These tubes are arranged in racks (Fig. 1). We have revised, restored, and fully databased this collection and all the tubes have been labelled adequately. Most of them do not have any identification, but bear adequate collection data. The records have been incorporated into MA-Algae databases and all the label details of the specimens are now available through the Internet at: http://www.rjb.csic.es/jardinbotanico/jardin/index. php?Cab $=109 \&$ SubCab=204\&len=en, http://datos.gbif.es $/$ collectory/public/showDataResource/dr90, and http://www. gbif.org/dataset/83459d16-f762-11e1-a439-00145eb45e9a.

A review of González Guerrero's botanical works was carried out and scientific names including publication and type details where databased using the computer application Bibmaster (Pando \& al., 2004). We have listed the names published by him in this paper. For each name, protologue - publication, type locality, and illustration details - are given, and figures are cited in this description. Type locality details have been literally transcribed.

\footnotetext{
* Corresponding author
} 


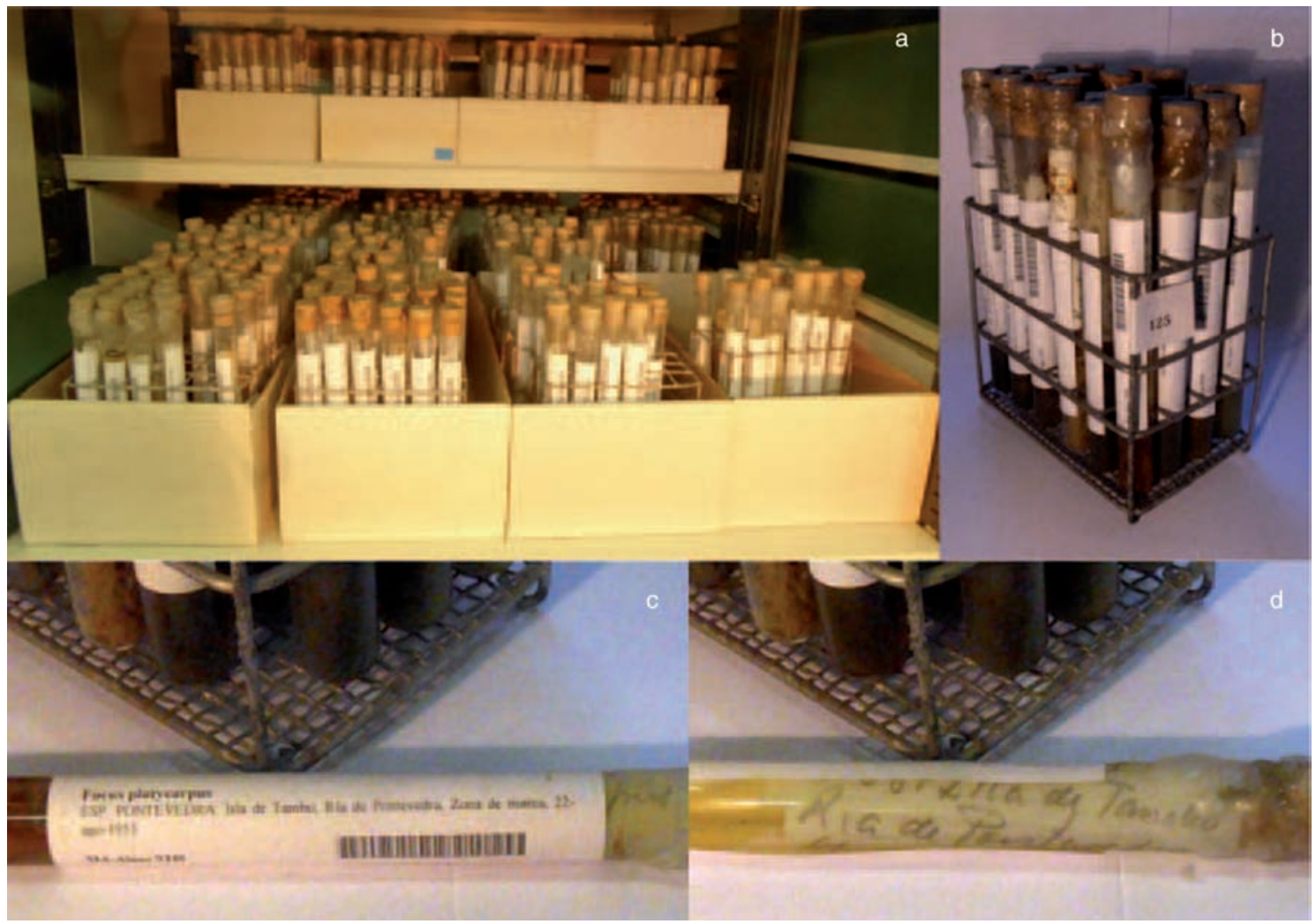

Fig. 1. González Guerrero's Algae Collection in its current location: a, partial view, arranged in shelves, boxes, and racks; b, rack; c, tube (MA-Algae 9340) showing label produced from the database; $\mathbf{d}$, tube (MA-Algae 9340) showing original label inside.

Protologue citation follows the TL-2 (Stafleu \& Cowan, 1976-1985; Stafleu \& Mennega, 1992-1998) and B-P-H (Lawrence \& al., 1968; Bridson \& Smith, 1991) abbreviations. When specimens which collection details matched those provided in the protologue have been found, their herbarium accession numbers have been included. These MA-Algae specimens constitute original material, most probably were used by González Guerrero in his original descriptions, and are therefore ideal material for lecto or epitypification purposes. In a few cases, dates shown on herbarium labels do not correspond exactly with those provided in the literature. In these instances, we discuss the possible cause of errors on tube labels or in publications. The list of published names is provided; we have included 3 names written on the herbarium labels but never published.

\section{RESULTS AND DISCUSION}

\section{The collection}

Currently, only 2155 specimens of the herbarium of the extinct Sección de Ficología remain preserved in formalin. The original collection was much larger, but it seems that a collapse in the old building, where the herbarium was kept, destroyed many tubes. Since the early 1970's, this collection has remained virtually untouched in the herbarium of the Royal Botanic Garden of Madrid. The history of the herbarium was briefly recorded by Gallardo \& al. (1993).

Most of the collection, 2003 specimens, was collected by P. González Guerrero between 1924 and 1974. Except for 15 tubes from Torrevieja -Alicante, Spain - and 7 tubes from Estoril - Portugal-, all the specimens are from a wide variety of continental water habitats - rivers, marshes, ponds, soil, thermal waters, and even monuments and buildings- . Although most of the samples are from the Iberian Peninsula (Fig. 2), a few samples were collected in other places -Argentina, Greece, Morocco, and Norway-. On the Iberian specimens, Cádiz province is the most represented - 822 tubes-. Other wellrepresented provinces are Madrid, Albacete, Ciudad Real, Badajoz, Granada, Huelva, Huesca, Lérida, Sevilla, Trasos-Montes, and Valencia. Regarding collectors, nearly all specimens were collected by González Guerrero, though Caballero, Cuatrecasas, Jordan de Urries, and Unamuno, among others, also collected some.

Only 92 tubes out of the total have identification down to the species level, being the usual case tubes with species mixtures with only indication of the better represented algae orders in the sample.

Among Gonzalez Guerrero's tubes, there are further 14 tubes containing sea algae collected by F. Miranda (19051964), the most relevant Spanish phycologist of the first half of the $20^{\text {th }}$ century (Dosil, 2001). Most of Miranda's original collections are kept in MAF herbarium - Universidad Complutense, Madrid-. However, materials from his Ph.D. Dissertation (1926-1928), as well as those from his sojourn in France (1931), were missing (Valenzuela \& Pérez-Cirera, 1982; Dosil, 2001). These authors mentioned that those materials might be kept either at the National Museum of Natural Sciences of Madrid or at the Royal 


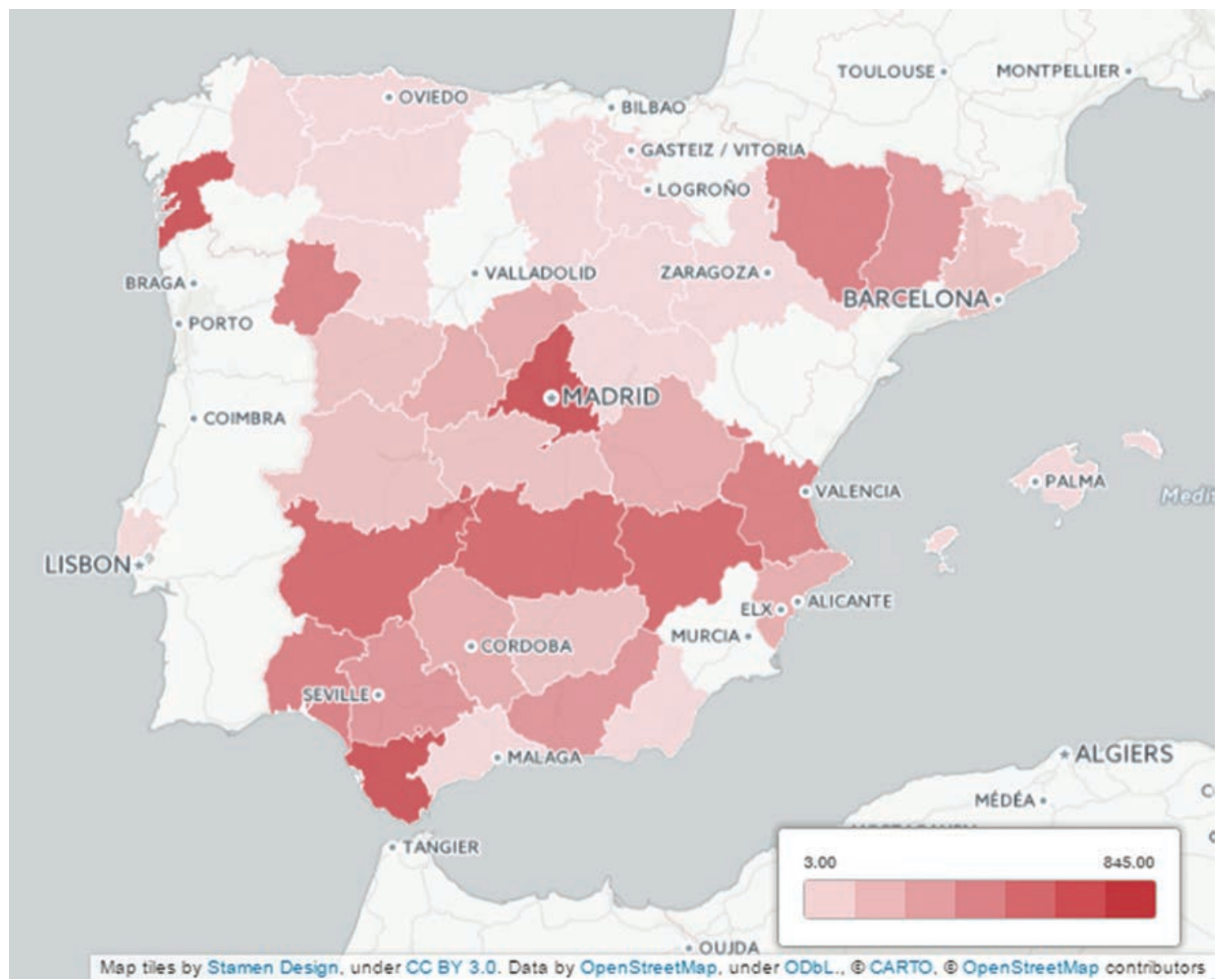

Fig. 2. Specimens collected by González Guerrero in the Iberian Peninsula grouped by provinces (Spain) and districts (Portugal).

Botanic Garden of Madrid. They looked for them unsuccessfully. The tubes mentioned above correspond to some of these missing specimens.

\section{Algae Names published by González Guerrero}

The following list comprises 70 cyanophytes, 28 chlorophytes, 4 xantophytes, 2 haptophytes, 1 crysophyte, and 1 dinoflagellate. When comparing the list with accounts and surveys of Iberian continental algae (Álvarez-Cobelas, 1984a, 1984b; Álvarez-Cobelas \& Gallardo, 1986, 1988; Cambra \& al., 1998), we observe that Gonzalez Guerrero's species are seldom cited, and their concept and circumscription problematic. We hope the information here presented to be an initial step for the clarification of these taxa and names, since the information available up to now, especially the illustrations, was often incomplete or too schematic to warrant solid taxonomic decisions. At the end of the list, 3 names found on the collection labels but never published are recorded. For each name protologue citation, original locality -ind. loc.-, and, in many cases, remarks about the name or a mention of the collection specimens which details match those of the protologue are included. We also uploaded to AlgaeBase (Guiry \& Guiry, 2016) those González Guerrero's names validly published and gathered here. Their details are available for download from that platform. No taxonomic or identification work was carried out in the context of this paper.

\section{Charophyta}

Cosmarium nitidulum f. gaditana P. González, Anales Jard. Bot. Madrid 7: 450, lám. 1 fig. 1 (1948)

Ind. loc.- "Vivit at fontes in loco dicto Parque del Genovés (Cádiz), legit. 2-I-1946."

Remarks.-MA-Algae 7711, MA-Algae 7718.

Pleurotaenium coronatum var. jorgensenum P. González, Anales Inst. Bot. Cavanilles 22: 171, lám. 1 fig. 3 (1965)

Ind. loc. - "Isla de los Osos, Noruega, agosto de 1961."

Remarks.-There are 48 specimens from Norway - August, 1951—, but none of them has indication of either "Isla de los Osos" or "Björnöya" localities.

\section{Chlorophyta}

Binuclearia palhinhae P. González, Agron. Lusit. 12: 62, lám. 1 fig. 1 (1950)

Ind. loc. - "Habitat in turfosis circa cacuminem «Altar de Cabrốes» dictum (1560 m. alt.) in Sierra de Gerês (Lusitania) ubi legi 3-VII-1948.” Remarks.-MA-Algae 7672 (July, 4th 1948).

Bumilleria fernandesii P. González, Agron. Lusit. 12: 74, lám. 3 fig. 20 (1950) 
Ind. loc.-_Vivit in aquis hyalinis versus fontibus fluminis Cávado (Lusitania) ubi legi VII-1948."

Remarks.-MA-Algae 9074, MA-Algae 9102.

Catena de-toniana P. González, Giorn. Bot. Ital. 71: 532, fig. 3 (1964)

Ind. loc.- "Legit: in aquis lente fluentibus, rivulus Valtournance, Monte Cervino (Matterhorn). 10-VIII-1958."

Chlamydomonas cavanillesiana P. González, Anales Jard. Bot. Madrid 6: 273, lám. 19 fig. 9 (1948)

Ind. loc._"Habitat in saliniis San Fernando (Cádiz). 2-IV-1944."

Gongrosira gaditana P. González, Anales Jard. Bot. Madrid 7: 447, lám. 3 fig. 41 (1948)

Ind. loc.- "Vivit in cochlea Lamellibranchiorum emortuorum in salinis prope San Fernando (Cádiz) ubi legi 3-I-1946.”

Remarks.-MA-Algae 7521, MA-Algae 7563, MA-Algae 7565, MA-Algae 7568, MA-Algae 7570, MA-Algae 7571, MA-Algae 7579, MA-Algae 7695, MA-Algae 7702, MA-Algae 7732, MA-Algae 7821, MA-Algae 7827, MA-Algae 7866, MA-Algae 7927, MA-Algae 7931, MA-Algae 7938.

Hormidium gaditanum P. González, Anales Jard. Bot. Madrid 7: 448, lám. 2 fig. 18 (1948)

Ind. loc. _ "In muris calcareis, San Fernando (Cádiz). 3-I-1946." Remarks. - There are 46 specimens collected on January, 3rd 1946 from that locality, but with no data about the habitat.

Kirchneriella secktiana P. González, Anales Jard. Bot. Madrid 1: 157, fig. 1.1 (1941)

Ind. loc._-"Algas de la República Argentina/.. /Habitat in aquis lente fluentibus prope Tanti, ubi 2-VII-1933 legi."

Lusitania P. González, Portugaliae Acta Biol., Sér. B, Sist. J. Henriques: 126, lám. 1 fig. 46 (1949)

Typus._Lusitania henriquesii P. González

Lusitania henriquesii P. González in Portugaliae Acta Biol., Sér. B, Sist. J. Henriques: 126, lám. 1 fig. 46 (1949)

Ind. loc.- "Planctonica vel plus minusve inmersa in aquiis fluminis Gerês cum Synedra ulna, Asterionella formosa, etc. Legi 4-VII-1948."

Remarks.-MA-Algae 9107.

Oedogonium de-tonii P. González, Giorn. Bot. Ital. 71: 528, fig. 1 (1964)

Ind. loc.- "Legit: ad folia Ranunculus et plantarum aliarum epiphyticum in aquis stagnantibus vel lente fluentibus. Arroyo del Ajo, Cabeza del Buey (Badajoz, España). 20-IV-1960.”

Oocystis rifeum P. González, Bol. Soc. Esp. Hist. Nat. 29: 253, figs. 9-12 (1929)

Ind. loc.- "Hab: in aquis stagnantibus in Telatza de Reixana, prope Larache (Marruecos) socia Scenedemus, Pediastrum, Spirogyra, Anabaenopsis, etcétera, 13-VII-923. Legit Prof. Caballero.”

Remarks.-MA-Algae 9030

Oocystis striata P. González, Anales Jard. Bot. Madrid 8: 272, lám. 1 fig. 14 (1949)
Ind. loc.- - In aquis stagnantibus in $<<$ Valle de la Vieja $>>$ dicto prope Castuera (Badajoz), legi 3-IV-1943."

Palmodictyon resendeanum P. González, Agron. Lusit. 12: 61, lám. 3 fig. 2 (1950)

Ind. loc._-"Vivit in turfosis prope Borrageira (Sierra de Gerês, Minium, Lusitania), VII-1948."

Remarks.-MA-Algae 7666 (July, 6th 1928).

Pediastrum conchense P. González, Anales Jard. Bot. Madrid 1: 119, fig. 5.15 (1941)

Ind. loc.- "Legit Prof. Caballero pago dicto $<<$ Puente Vadillos $>>$ prope, Cañizares (Cuenca) VI-1935."

Pediastrum contiguum P. González, Anales Jard. Bot. Madrid 1: 148, fig. 1.9 (1941)

Ind. loc.- "Algas de la República Argentina/.. /Habitat in aquis stagnantibus et lente fluentibus. Tanti, VII-1933 legi."

Pediastrum tetrax var. octogonis P. González, Bol. Soc. Esp. Hist. Nat. 29: 252, figs. 7-8 (1929)

Ind. loc.- "Habita con otras Protococcáceas (Oocystis, Scenedesmus, etc.) y algas filamentosas (Oedogonium, Bulbochaete, Spirogyra, etc.) en los charcos de Telatza de Reixana, cerca de Larache (Marruecos), 13-VII923. Legit Prof. Caballero."

Remarks.-MA-Algae 9030

Placosphaera cavanillesiana P. González, Anales Jard. Bot. Madrid 6: 271, lám. 21 fig. 1 (1948)

Ind. loc.- "Habitat in parietibus calcareis emanationibus sulphidricis submissis balneario de Fuente Amarga prope Chiclana de la Frontera (Cádiz). 3-IV-1944."

Remarks.-MA-Algae 7964, MA-Algae 7965, MA-Algae 7966 , MA-Algae 7971.

Protoderma cavanillesiana P. González, Anales Jard. Bot. Madrid 6: 276, lám. 20 fig. 3 (1948)

Ind. loc.- "Vivit supra Lolam lubricam in saliniis prope San Fernando (Cádiz). 2-IV-1944."

Protoderma pax-augustana P. González, Anales Jard. Bot. Madrid 8: 274, lám. 2 fig. 1 (1949)

Ind. loc.- "Habitat supra concham Lamellibranchiae submersae sessilis in parietate calcarea aquarii $<<$ Pilar nuevo $>>$ dicto prope Esparragosa de Lares (Badajoz) ubi legi 2-I-1948."

Scenedesmus aciculatus P. González, Anales Jard. Bot. Madrid 1: 156, figs. 2.21, 2.21 bis (1941)

Ind. loc._- "Algas de la República Argentina/../In rivulis. Tanti, VII-1933."

Scenedesmus heteracanthus P. González, Anales Jard. Bot. Madrid 1: 154, fig. 1.8 (1941)

Ind. loc.—-Algas de la República Argentina/.. /Habitat in aquis lente fluentibus prope Tanti; legi VII-1933."

Scenedesmus longus var. carpetana P. González, Bol. Soc. Esp. Hist. Nat. 29: 254, figs. 13-16 (1929) 
Ind. loc.- "Habita con los géneros Tetmemorus, Pediastrum, Sorastrum, Micrasterias, Synedra, etc., en la Laguna de Peñalara (Madrid), 6-VIII-925."

Remarks.-MA-Algae 8443, MA-Algae 8449, MA-Algae 8450, MA-Algae 8468, MA-Algae 8469.

Scenedesmus verrucosus P. González, Anales Jard. Bot. Madrid 1: 153, fig. 2.19 (1941)

Ind. loc.- "Algas de la República Argentina/../ Habitat in aquis lente fluentibus prope Tanti, ubi VII-1933 legi."

Stichococcus castellanus P. González, Anales Jard. Bot. Madrid 8: 273, lám. 2 fig. 3 (1949)

Ind. loc.- "Vivit in testa culturae in hiberna Horti Botanici matritensi ubi legi 3-IV-1948."

Tetraedron muticum f. hispanica P. González, Anales Jard. Bot. Madrid 8: 273, lám. 2 fig. 8 (1949), nom. nud.

Ind. loc.- "Vive planctónica entre otras algas unicelulares o filamentosas, en La Serena (Castuera, Badajoz). IV-1943."

Tetrastrum glabrum var. hispanica P. González, Anales Jard. Bot. Madrid 8: 273, lám. 2 fig. 14 (1949)

Ind. loc.- "Vivit planctonico in aquis stagnantibus in loco dicto $<<$ Valle de la Vieja >>, prope Castuera (Badajoz), ubi legi 3-IV-1944."

\section{Cyanobacteria}

Anabaena cavanillesiana P. González, Anales Jard. Bot. Madrid 6: 258, lám. 6 fig. 6 (1948)

Ind. loc.- "Habitat libere natante vel in fundo salinarum prope Puerto Real (Cádiz). 3-IV-1944 ubi legi."

Remarks.-MA-Algae 7705.

Anabaena pintoi P. González, Agron. Lusit. 12: 59, lám. 1 fig. 14 (1950)

Ind. loc.- "Hab. planctonica in aquiis turfosis ad primam fluminis Homem in Serra de Gerês (Lusitania) $1550 \mathrm{~m}$ alt. ubi legi 3-VII-1948." Remarks.-MA-Algae 7670, MA-Algae 7674.

Anabaena scheremetievi var. ifniensis P. González, Anales Jard. Bot. Madrid 1: 101, fig. 7 (1941)

Ind. loc.-_En aguas salobres del río Ifni (África occidental), junio-julio de 1934. Legit Prof. Caballero."

Remarks.-MA-Algae 9026, MA-Algae 9029.

Anabaenopsis cuatrecasasii P. González, Bol. Soc. Esp. Hist. Nat. 28: 436, figs. 4-6 (1928)

Ind. loc.- "Hab.: in paludosis prope Baños de Montemayor (Cáceres), IX-1927. Legit Prof. Caballero. Apud fontem Bedmar (Jaen) 2-VI-1928. Legit Prof. Cuatrecasas."

Remarks.-MA-Algae 8148, MA-Algae 8151, MA-Algae 8154 [Cáceres]. Later synonymized to Cylindrospermum majus Kütz. (GonzálezGuerrero, 1931).

Anabaenopsis hispanica P. González, Bol. Soc. Esp. Hist. Nat. 28: 358, figs. 1-3 (1928)

Ind. loc.- "Habitat in stagnis prope Baños de Montemayor (Cáceres), ubi legit Prof. A. Caballero, IX-927."
Remarks.-MA-Algae 8148, MA-Algae 8151, MA-Algae 8154. Later synonymized with Cylindrospermum licheniforme (Bory) Kütz. (GonzálezGuerrero, 1943).

Anabaenopsis hispanica var. luteola P. González, Bol. Soc. Esp. Hist. Nat. 28: 359 (1928)

Ind. loc.- "In stagnis Horti Botanici Matritensis, X-1924."

Remarks.-Later synonymized with Cylindrospermum licheniforme (Bory) Kütz. (González-Guerrero, 1943).

Arthrodesmus incus var. juressi P. González, Agron. Lusit. 12: 71, lám. 2 fỉg. 42 (1950)

Ind. loc. - "In humo prope cuspidum Borrageira. VII-1948."

Remarks.-MA-Algae 7666 [The wording of the label matches exactly the wording of the publication except for the fact that the date reads "6-VII-1928" instead of "VII-1948"].

Arturoa P. González, Anales Jard. Bot. Madrid 10: 210, lám. 2 fig. 1 (1952)

Typus.-Arturoa anae P. González.

Arturoa anae P. González, Anales Jard. Bot. Madrid 10: 211, lám. 2 fig. 1 (1952)

Ind. loc.- "Habitat: in terreno argilloso in aquis salsis de Ayamonte-Isla Cristina etiam in fluminibus Odiel et Guadalete./.. /VI-1950."

Aulosira godoyana P. González, Anales Jard. Bot. Madrid 8: 270, lám. 2 fig. 15 (1949)

Ind. loc.- - In aquis lentefluentibus in rivis $<<$ Valle de la Vieja $>>$ dicto prope Castuera (Badajoz), ubi legi 3-IV-1943."

Calothrix candelii P. González, Bol. Soc. Esp. Hist. Nat. 30: 413, figs. 2-5 (1930)

Ind. loc.- "Hab.: ad rivulos Zauía Muley Bagdad (Melilla), 27-X-929. Legit Prof. Candel”

Chamaesiphon duran-mileri P. González, Anales Jard. Bot. Madrid 6: 249, lám. 13 fig. 6 (1948)

Ind. loc.- "In salinis Puerto Real dicto (Cádiz) vivit epiphyta supra Lolam lubricam 3-IV-1944 legi."

Remarks.-MA-Algae 7705

Chroococcus lithophilus var. rotae P. González, Anales Jard. Bot. Madrid 8: 266, lám. 1 fig. 9 (1949)

Ind. loc.- "Vivit supra parietatem calcaream submersum prope Rota (Gades) ubi legi 5-X-1945."

Chroococcus mutisii P. González, Anales Jard. Bot. Madrid 7: 436, lám. 2 fig. 27 (1948)

Ind. loc.- "Habitat in flumine Arillo prope San Fernando (Gadir) ubi legi 3-I-1946."

Remarks.-MA-Algae 7943, MA-Algae 7567 (?), MA-Algae 8046 (?), MA-Algae 8049 (?).

Chroococcus tenax var. boeticus P. González, Anales Jard. Bot. Madrid 6: 242, lám. 6 fig. 1 (1948)

Ind. loc.- "Vivit in salinis gaditanis ubi legi 2-IV-1944."

Remarks.-MA-Algae 9111, MA-Algae 9112, MA-Algae 9114 MA-Algae 9131. 
Cyanothrix cavanillesii P. González, Anales Jard. Bot. Madrid 6: 250, lám. 4 fig. 4 (1948)

Ind. loc.- "Habitat in lutosis prope Puerto de Santa María (Cádiz) ad ostium fluminis Guadalete ubi legi 1-IV-1944."

Remarks.-MA-Algae 7736, MA-Algae 7742, MA-Algae 7968, MA-Algae 9121.

Dermocarpa aquae-dulcis var. geresiana P. González in Agron. Lusit. 12: 47, lám. 3 fig. 23 (1950)

Ind. loc.- "In Scytonema, in foliis submersis, etc., prope Bouça da Mó, in flume Homem. Serra do Gerês. VII-1948."

Remarks.-MA-Algae 7675, MA-Algae 9048.

Dermocarpa cavanillesiana P. González, Anales Jard. Bot. Madrid 6: 249, lám. 20 fig. 2 (1948)

Ind. loc.- "In flumine Arillo vivit supra Lolam lubricam prope San Fernando (Cádiz). 4-IV-1944 legi."

Remarks.-MA-Algae 7703, MA-Algae 7710, MA-Algae 7716, MA-Algae 9123, MA-Algae 9128.

Dermocarpa gaditana P. González, Anales Jard. Bot. Madrid 7: 442, lám 3 fig. 35 (1948)

Ind. loc.- "Vivit perforantem in cochlea Gasteropodum vivum in flumine Arillo circa San Fernando (Cádiz) ubi legi 3-I-1946."

Remarks.-MA-Algae 7943, MA-Algae 7567 (?), MA-Algae 8046 (?), MA-Algae 8049 (?).

Dermocarpa versicolor var. lusitana P. González, Agron. Lusit. 12: 48, lám. 1 fig. 9 (1950)

Ind. loc.- "Hab.: in aliis algis intermissa, in flume Homem, VII-1948." Remarks.-MA-Algae 7670, MA-Algae 7674, MA-Algae 9045, MA-Algae 9047.

Dichothrix catalaunica P. González, Cavanillesia 3: 55, figs. $1-5(1930)$

Ind. loc.- "Hab.: in parietibus cujusdam putei Universitatis Barcinonensis; XII-928, cl. Prof. Cuatrecasas legit."

Dichothrix cavanillesii P. González, Anales Jard. Bot. Madrid 6: 251, lám. 8 fig. 3, láms. 9-10, fig. 1 (1948)

Ind. loc.- "Vivit in lutosis salinarum Puerto Real (Cádiz) ubi legi 3-IV-1944."

Remarks.-MA-Algae 7705.

Eucapsis salina P. González, Anales Jard. Bot. Madrid 8: 267, lám. 1 fig. 1 (1949)

Ind. loc.- "Habitat planktonica in aquis maritimis prope San Fernando (Gadir) ubi legi 3-I-1946."

Remarks. - We point out here the fact that the foot of lam. 1 fig. 1 reads "Eucapsis alpina". There are 46 specimens collected on Jaunary, 3rd 1946 from that locality, but with no data about the habitat.

Gloeotrichia natans var. zujaris P. González, Bol. Soc. Esp. Hist. Nat. 30: 223, figs. 1-6 (1930)

Ind. loc.—"Hab.: in foliis caulibusque Myriophylli sp./../ $<<$ Vado de los Pontones $>>$, río Zujar, Esparragosa de Lares (Badajoz), 10-X-1926 y a medio metro de profundidad.” [sec. P. González, 1930: 227].

Remarks.-Later considered a development stage of the typical form (González-Guerrero, 1943).
Holopedia clementei P. González, Anales Jard. Bot. Madrid 6: 243, lám. 4 fig. 5 (1948)

Ind. loc.- "In stagnis marginalibus fluminis Guadalete dicto prope Puerto de Santa María (Gades) legi 3-IV-1944."

Remarks.-MA-Algae 7830 (April, 2nd 1944), MA-Algae 9366 (April, 1st 1944).

Holopedia mutisiana P. González, Anales Jard. Bot. Madrid 6: 243, lám. 4 fig. 6 (1948)

Ind. loc.- "In stagnis ostii fluminis San Pedro dicto prope Gades. 1-IV-1944." Remarks.-MA-Algae 7484, MA-Algae 7758, MA-Algae 7795 , MA-Algae 7803, MA-Algae 7809, MA-Algae 7961, MA-Algae 7963, MA-Algae 7970, MA-Algae 7972; additionally MA-Algae 9126 from "Desembocadura río Guadalete, Puerto de Santa María (Ca), 2 April 1944" is identified as "Holopedia mutisiana".

Homoeothrix caespitosa var. argentinensis P. González, Anales Jard. Bot. Madrid 5: 331, láms. 1-3 (1946)

Ind. loc.- "Habitat in aquis fluentibus prope Saldan [(Córdoba). República Argentina]. VI-1933, legi."

Hormathonema onychoides P. González, Anales Jard. Bot. Madrid 10: 202, lám. 1 fig. 6 (1952)

Ind. loc.- "In petris calcariis litoris de Torrevieja (Alicante), 3 julio 1950." Remarks.-MA-Algae 7377, MA-Algae 8707, MA-Algae 8709, MA-Algae 8710, MA-Algae 8711, MA-Algae 8713, MA-Algae 8714, MA-Algae 8715, MA-Algae 9371.

Hydrocoleus cavanillesii P. González, Anales Jard. Bot. Madrid 6: 270, lám. 5 fig. 3 (1948)

Ind. loc.—“Hab. in salinis Puerto Real (Cádiz). 2-IV-1944."

Remarks-MA-Algae 9111, MA-Algae 9112, MA-Algae 9114, MA-Algae 9131.

Isocystis andalusicus P. González, Anales Jard. Bot. Madrid 6: 259, lám. 6 fig. 5 (1948)

Ind. loc.- "Vivit endophytica in foliis Phanerogamarum inmersarum in salinis prope Puerto Real (Cádiz). 4-IV-1944 ubi legi."

Lyngbya aestuarii var. gaditana P. González, Anales Jard. Bot. Madrid 7: 445, lám. 2 fig. 24 (1948)

Ind. loc.- "Habitat in solo salso fluminis San Pedro prope Puerto de Santa María; legi 26-XII-1945."

Remarks.-MA-Algae 7335, MA-Algae 7337, MA-Algae 7339, MA-Algae 7340, MA-Algae 7341, MA-Algae 7342, MA-Algae 7345, MA-Algae 7346, MA-Algae 7348, MA-Algae 7349, MA-Algae 7354, MA-Algae 7355, MA-Algae 7356, MA-Algae 7358, MA-Algae 7359, MA-Algae 7360.

Lyngbya lagerheimii var. crustacea P. González, Anales Jard. Bot. Madrid 6: 266, lám. 2 fig. 1 (1948), nom. nud.

Ind. loc.- "San Fernando 4-IV-1944. Adheridos a la parte inferior de un tubo de hierro que conduce el agua potable a Cádiz sobre el río Arillo." Remarks.-MA-Algae 7709.

Microchaete setcasasii P. González, Bol. Soc. Esp. Hist. Nat. 30: 412, fig. 1 (1930)

Ind. loc.- "Hab.: ad rupes in aquis inmersas, prope Setcasas (Gerona), 29-VIII-929."

Remarks.-MA-Algae 8997 (August, 28th 1928). 
Microcoleus cavanillesii P. González, Anales Jard. Bot. Madrid 6: 270, lám. 7 fig. 2 (1948)

Ind. loc._-"Habitat in fluminibus Iro, San Pedro, Guadalete, etc. (Cádiz). 4-IV-1944."

Remarks.-MA-Algae 7730 (August, 3rd 1944).

Microcystis parasitica var. geresiana P. González, Agron. Lusit. 12: 37, lám. 3 fig. 7 (1950)

Ind. loc.- "In aquis turfosis prope Borrageira, VII-1948."

Remarks.-MA-Algae 7666 (July, 6th 1928).

Montanoa P. González, Anales Jard. Bot. Madrid 8: 267, lám. 2 fig. 4 (1949)

Typus._Montanoa castellana P. González

Montanoa castellana P. González, Anales Jard. Bot. Madrid 8: 268, lám. 2 fig. 4 (1949)

Ind. loc.-_In testa culturae in Horto Botanico Matriti legi 3-IV-1948."

Nematoradaisia gasteropodum P. González, Anales Jard. Bot. Madrid 7: 438, lám. 3 fig. 34 (1948)

Ind. loc.- "Habitat perforantem in cochleis Gasteropodum vivorum in flumine Arillo prope San Fernando (Cádiz) ubi legi 3-I-1946."

Remarks.-MA-Algae 7943, MA-Algae 7567 (?), MA-Algae 8046 (?), MA-Algae 8049 (?).

Nodularia skujae P. González, Bol. Soc. Esp. Hist. Nat. 28: 435, figs. 1-3 (1928)

Ind. loc.- "Hab.: in ulceribus Ulmi campestris in loco dicto Casa de Campo, 7-VI-1928, et in ulceribus Populi sp. Vaciamadrid (Madrid) 2-V-1928."

Remarks.-MA-Algae 8537 ("Casa de Campo"), MA-Algae 8515 ("Vaciamadrid").

Nodularia spumigena var. zujaris P. González, Bol. Soc. Esp. Hist. Nat. 30: 224, figs. 7-8 (1930)

Ind. loc.-"Hab.: ad Gloeotrichiam natantem Rab. varietatis zujaris./../ $<<$ Vado de los Pontones $>>$, río Zujar, Esparragosa de Lares (Badajoz), 10-X-1926 y a medio metro de profundidad” (sec. P. González, 1930: 227).

Nostoc halophilum var. gaditanum P. González, Anales Jard. Bot. Madrid 7: 443, lám. 1 fig. 12 (1948)

Ind. loc. - "Habitat ad terram plus minusve humidam in marginibus salinarum prope San Fernando (Cádiz). 3-I-1946.”

Remarks.-MA-Algae 7707.

Oscillatoria cavanillesiana P. González, Anales Jard. Bot. Madrid 6: 264, lám. 3 fig. 4 (1948)

Ind. loc.-"Vivit in aquiis salsis istmi gaditani ubi legi 4-IV-1944."

Remarks.-MA-Algae 7466, MA-Algae 7468, MA-Algae 7473, MA-Algae 7474, MA-Algae 7476, MA-Algae 7480, MA-Algae 7762, MA-Algae 7802, MA-Algae 7804, MA-Algae 7806, MA-Algae 7807, MA-Algae 7808, MA-Algae 7810, MA-Algae 7813, MA-Algae 8025, MA-Algae 9120

Oscillatoria minutissima P. González, Anales Jard. Bot. Madrid 7: 445, lám. 2 fig. 19 (1948)

Ind. loc._-"Habitat in muris calcareis in San Fernando (Cádiz). 3-I-1946."
Remarks. - There are 46 specimens collected on Jaunary, 3rd 1946 from that locality, but with no data about the habitat.

Oscillatoria salina var. boetica P. González, Anales Jard. Bot. Madrid 7: 445, lám. 2 fig. 25 (1948)

Ind. loc.- "Habitat in solo salso in marginibus fluminis San Pedro prope Puerto de Santa María (Cádiz) ubi legi. 26-XII-1946 [sic]."

Remarks.-MA-Algae 7335, MA-Algae 7337, MA-Algae 7339 , MA-Algae 7340, MA-Algae 7341, MA-Algae 7342, MA-Algae 7345, MA-Algae 7346, MA-Algae 7348, MA-Algae 7349, MA-Algae 7354, MA-Algae 7355, MA-Algae 7356, MA-Algae 7358, MA-Algae 7359, MA-Algae 7360, MA-Algae 7361, MA-Algae 7365, MA-Algae 7366, MA-Algae 7367, MA-Algae 7368, MA-Algae 7374, MA-Algae 7502, MA-Algae 7573, MA-Algae 7861, MA-Algae 7867, MA-Algae 7872, MA-Algae 7874, MA-Algae 7876, MA-Algae 7877.

The date of the protologue is probably a mistake and should be "26XII-1945". In the introduction of the paper, González stated that all the cited specimens were collected during the 1945/46 winter, which agrees with the label's date and the remaining specimens studied in the paper.

Oscillatoria willei var. valentina P. González, Anales Jard. Bot. Madrid 5: 310, lám. 2 fig. 3 (1946)

Ind. loc.- "Vive en una acequia con escasa cantidad de agua detenida Chirivella. Valencia. 2-III-1938. Legit Prof. Mendigutia."

Phythelios de-tonii P. González, Giorn. Bot. Ital. 71: 535, fig. 5 (1964)

Ind. loc.- "Legit: in aquis stagnantibus, vel lente fluentibus in rivulo Guadarrama (Galapagar, Madrid). Agosto 1959."

Polychlamydum cavanillesii P. González, Anales Jard. Bot. Madrid 6: 265, lám. 3 fig. 1 (1948)

Ind. loc. - "Vivit in omnia regione salinaria gaditana ubi legit 2-IV-1944." Remarks.-MA-Algae 9111, MA-Algae 9112, MA-Algae 9114 MA-Algae 9131.

Rhabdoderma cavanillesiana P. González, Anales Jard. Bot. Madrid 6: 244, lám. 4 fig. 8 (1948)

Ind. loc.- "In salinis prope ostii fluminis San Pedro legi 1-IV-44." Remarks.-MA-Algae 7482, MA-Algae 7706.

Rivularia beccariana var. de-toniana P. González, Giorn. Bot. Ital. 71: 538, fig. 7, 8 (1964)

Ind. loc.- "Legit: in rivulo Guadiana. Ruidera (Ciudad Real). 28-VI-1956."

Remarks.-MA-Algae 8254, MA-Algae 8975, MA-Algae 8976 (labelled "28-VI-1954").

Rivularia cavanillesiana P. González, Anales Jard. Bot. Madrid 6: 255, lám. 25 fig. 2 (1948)

Ind. loc.- "In ligno inmerso flumine Arillo vivit prope San Fernando (Cádiz). 4-IV-1944."

Remarks.-MA-Algae 7703, MA-Algae 7710, MA-Algae 7716, MA-Algae 9123, MA-Algae 9128.

Sampaionema P. González, Agron. Lusit. 12: 50, lám. 3 fig. 3 (1950)

Typus._Sampaionema lacerdana P. González

Sampaionema lacerdana P. González, Agron. Lusit. 12: 51, lám. 3 fig. 3 (1950) 
Ind. loc._-"Vivit cum Tribonema ulotricoide in turfosis prope Borrageira, ubi legi VII-1948."

Remarks.-MA-Algae 7666 (July, 6th 1928).

Schizothrix cavanillesii P. González, Anales Jard. Bot. Madrid 6: 268, lám. 16 fig. 1 (1948)

Ind. loc._-"Vivit in flumine Arillo (Cádiz) ubi legi 4-IV-1944."

Remarks.-MA-Algae 7703, MA-Algae 7710, MA-Algae 7716 , MA-Algae 9123, MA-Algae 9128.

Scytonema andradeana P. González, Agron. Lusit. 12: 58, lám. 4 fig. 2 (1950)

Ind. loc._-"En las aguas turbosas del pico de la Borrageira."

Remarks.-MA-Algae 7666 (July, 6th 1928).

Scytonema cuatrecasasii P. González, Cavanillesia 3: 56, figs. 6-9 (1930)

Ind. loc.- "Hab.: in rupibus irroratis, prope Molí de Sau, Les Guilleries (Catalaunia), 4-XI-929. Legit Prof. Cuatrecasas.”

Spelaeopogon populi P. González, Mem. Real Soc. Esp. Hist. Nat. 15: 437, figs. 1-13 (1929)

Ind. loc._-"Hab.: Socia Nodulariae skujae Gonz. Guerr., in ulceribus Populi sp. Vaciamadrid (Madrid), 2-V-928."

Remarks.-MA-Algae 8515.

Spirulina breviarticulata var. anguiformis P. González, Anales Jard. Bot. Madrid 6: 260, lám. 7 fig. 1 (1948)

Ind. loc.- "Vivit in marginibus fluminis San Pedro, prope Cádiz. 1-IV-1944."

Remarks.-MA-Algae 7739

Spirulina cabrerae P. González, Anales Jard. Bot. Madrid 6: 262, lám. 8 fỉg. 1 (1948)

Ind. loc. - "Vivit plancktonica in flumine Iro prope Chiclana de la Frontera (Cádiz). 3-IV-1934 [sic]." In this paler, below, González indicated "3-IV-1944", which agrees with the extant herbarium material and other specimens cited in the paper.

Remarks.-MA-Algae 7730.

Spirulina cavanillesiana P. González, Anales Jard. Bot. Madrid 6: 262, lám. 8 fig. 2 (1948)

Ind. loc.- "Vivit in limo salinarum Puerto Real (Cádiz). 2-IV-1944."

Remarks.-MA-Algae 9111, MA-Algae 9112, MA-Algae 9114, MA-Algae 9131.

Spirulina gordiana P. González, Anales Jard. Bot. Madrid 6: 261, lám. 5 fig. 2; lám. 14, fig. 1 (1948)

Ind. loc.- "Habitat in fundo salinis et in ostio fluminis San Pedro (Cádiz). 1-IV-1944."

Remarks.-MA-Algae 7795 (identified as such on the label), MA-Algae 7482, MA-Algae 7706, MA-Algae 7963, MA-Algae 7970.

Staurocladia P. González, Anales Inst. Bot. Cavanilles 23: 135, lám. 1 fig. B (1968)

Typus._Staurocladia carpetana P. González

Staurocladia carpetana P. González, Anales Inst. Bot. Cavanilles 23: 136, lám. 1 fig. B (1968)
Ind. loc. - "Hab.: in aquis caenosis, siliceis, stagnantibus vel lente fluentibus, prope lacu Peñalara, 2200 ms. alto, Montis Carpetanus (Madrid). 6-VIII-1925."

Remarks.-MA-Algae 8443, MA-Algae 8449, MA-Algae 8450, MA-Algae 8468, MA-Algae 8469.

Tolypothrix cavanillesiana P. González, Anales Jard. Bot. Madrid 5: 313, lám. 1 fig. 4 (1946)

Ind. loc.- "Habitat in stagnis Horti Botanici Valentini. Valentia. 7-V1938. Legit González Guerrero."

Remarks.-MA-Algae 8606, MA-Algae 8607.

Wollea lemnae P. González, Anales Jard. Bot. Madrid 8: 268, lám. 1 fig. 2 (1949)

Ind. loc.- "Habitat endophytica in parenchyma dejecto foliorum Lemnae trisulcae in aquis salsis lacunae $<<$ Guarramar $>>$ circa Sanlúcar de Barrameda (Gadir) ubi legi 29-XII-1946."

Remarks.-MA-Algae 7995, MA-Algae 8005, MA-Algae 8008.

Xenococcus gaditanus P. González, Anales Jard. Bot. Madrid 7: 438, lám. 3 fig. 40 (1948)

Ind. loc.- "Habitat in cochlaea Gasteropodum vivum in loco dicto. Salinas de San Fernando (Cádiz) ubi legi 3-I-1946.”

\section{Haptophyta}

Calyptrosphaera glabra P. González, Anales Jard. Bot. Madrid 8: 279, lám. 2 fig. 27 (1949)

Ind. loc.- "Vivit natante in aquis salsis de $<<$ Guarramar $>>$ prope Sanlúcar de Barrameda (Cádiz) ubi legi 2-I-1946."

Calyptrosphaera sanlucari P. González, Anales Jard. Bot. Madrid 8: 279, lám. 2 fig. 26 (1949)

Ind. loc.- "Vivit natante in lacuna salsa $<<$ Guarramar $>>$ dicta prope Sanlúcar de Barrameda (Cádiz) ubi legi 2-I-1946.”

\section{Miozoa}

Exuviaella cavanillesiana P. González, Anales Jard. Bot. Madrid 6: 279, lám 21 fig. 8 (1948)

Ind. loc.- "Habitat in stagnis ab marginibus fluminis Guadalete, prope Puerto de Santa María (Cádiz). 2-IV-1944."

Remarks.-MA-Algae 7830.

\section{Ochrophyta}

Stokesiella cavanillesiana P. González, Anales Jard. Bot. Madrid 6: 278, lám. 19 fig. 8 (1948)

Ind. loc.- "Vivit in flumine Guadalete prope Puerto de Santa María. (Cádiz). 4-IV-1944."

Vaucheria micranthera P. González, Anales Jard. Bot. Madrid 5: 317, lám. 2 fig. 1 (1946)

Ind. loc.- "Habitat in fossis fluvii Turia. Valencia. 26-III-1938. Legit González Guerrero.”

Remarks.-MA-Algae 8649. 
Vaucheria pachyderma var. cerebrina P. González, Bol. Soc. Esp. Hist. Nat. 31: 638, fig. 8 (1931)

Ind. loc.-"En las aguas colgantes, Artá (Mallorca), 25-III-929.”

\section{Rhodophyta}

Aspalatia andalousica P. González, Anales Jard. Bot. Madrid 7: 438, lám. 3 fig. 33 (1948)

Ind. loc.- "Habitat perforantem in cochleis Gasteropodum vivorum in flumine Arillo prope San Fernando (Cádiz) ubi legi 3-I-1946."

Remarks.-MA-Algae 7943, MA-Algae 7567(?), MA-Algae 8046(?), MA-Algae 8049 (?).

Myxohiella cavanillesiana P. González, Anales Jard. Bot. Madrid 6(1): 247, lám. 18 fig. 1 (1948)

Ind. loc.- "Habitat endophytica foliis Fanerogamarum inmersarum in salinis fluminis Arillo prope San Fernando (Gades) ubi legi 4-IV-944."

Remarks.-MA-Algae 7703, MA-Algae 7710, MA-Algae 7716, MA-Algae 9123, MA-Algae 9128.

\section{Incerta sedis}

Marssoniella carpetana P. González, Bol. Soc. Esp. Hist. Nat. 29: 251, figs. 1-3 (1929)

Ind. loc.- "Hab.: in lacu dicto Peñalara (Madrid), 6-VIII-925."

Remarks.-MA-Algae 8443, MA-Algae 8449, MA-Algae 8450, MA-Algae 8468, MA-Algae 8469.

Peronyella pandorinae P. González, Anales Jard. Bot. Madrid 8: 278, lám. 1 fig. 4 (1949)

Ind. loc.- "Habitat in gelatina Pandorinae Mori in aquis aliquid salsis lacunae <<Guarramar $>>$ prope Sanlucar de Barramedo (Cádiz) ubi legi 20-XII-1946."

\section{Unpublished names and undescribed taxa}

\section{Microcoleus evagineus P. González, nom. inéd.}

Label data.- "Ayamonte, Huelva, 27-VI-1949."

Remarks.-MA-Algae 8804.

\section{Polychlamydum gaditanum P. González, nom. inéd.}

Label data._- “[ESP. Ca] En las salinas de la finca «el violín », San Fernando, Cádiz, 2-V-1944.”

Remarks.-MA-Algae 7577.

\section{Synechococcus sp.}

Label data.- "Pennales, Zygnemales, Synechococcus ? sp. nov. [ESP.Gr] Mulhacén, 12-VIII-1964, Terreno turboso, P. González Guerrero" Remarks.-MA-Algae 8913.

\section{ACKNOWLEDGEMENTS}

The authors thank Dr. Tomás Gallardo (Madrid) for the critical reading of the manuscript and his valuable suggestions.

\section{REFERENCES}

Álvarez-Cobelas, M. 1984a. Catálogo de las algas continentales españolas. II. Craspedophyceae, Cryptophyceae, Chrysophyceae, Dinophyceae, Euglenophyceae, Haptophyceae, Phaeophyceae, Rhodophyceae, Xanthophyceae. Acta Botanica Malacitana 9: 27-40.

Álvarez-Cobelas, M. 1984b. Catálogo de las algas continentales españolas. III. Zygophyceae Widder 1960. Collectanea Botanica 15: 17-37.

Álvarez-Cobelas, M. \& Gallardo, T. 1985. In memoriam. Pedro González Guerrero. Anales Jardín Botánico de Madrid 42: 3-7.

Álvarez-Cobelas, M. \& Gallardo, T. 1986. Catálogo de las algas continentales españolas. IV. Chlorophyceae Wille in Warming 1884 y Prasinophyceae T. Christensen ex Silva 1980. Acta Botanica Malacitana 11: 17-38.

Álvarez-Cobelas, M. \& Gallardo, T. 1988. Catálogo de las algas continentales españolas. V. Cyanophyceae Schaffner 1909. Acta Botanica Malacitana 13: 53-76.

Bridson, G.D.R. \& Smith, E.R. 1991. Botanico-periodicum-huntianum/ supplementum. Hunt Institute for Botanical Documentation, Pittsburgh.

Cambra, J., Álvarez-Cobelas, M. \& Aboal, M. 1998. Lista florística y bibliográfica de los clorófitos (Chlorophyta) de la Península Ibérica, Islas Baleares e Islas Canarias. (Listas de la flora y fauna de las aguas continentales de la Península Ibérica.) Asociación Española de Limnología, vol. 14.

Dosil, J. 2001. El estudio de las algas marinas en España: de la catalogación florística al aprovechamiento industrial (1814-1939). Tesis Doctoral, Universidad de la Coruña, Spain.

Gallardo, T., Margalet, J.L. \& Pérez-Ruzafa, I. 1993. Las colecciones históricas de algas españolas. In: Palacios, F., Martínez, C. \& Thomas, B. (eds.). Contributions on the status, preservation, and conservation of natural history collections. (Simposio Internacional y Primer Congreso Mundial sobre Preservación y Conservación de Colecciones de Historia Natural.) Ministerio de Cultura, Madrid.

González Guerrero, P. 1928. Más datos ficológicos de agua dulce. Boletín de la Sociedad Española de Historia Natural 28: 435-438.

González Guerrero, P. 1928. El género Anabaenopsis (Wolosz.) V. Miller en España. Boletín de la Sociedad Española de Historia Natural 28: 357-359.

González Guerrero, P. 1929. El género Spelaeopogon Borzi en España. Memorias de la Real Sociedad Española de Historia Natural 15: 437-439.

González Guerrero, P. 1929. Nuevos datos de plancton hispano-marroquí (agua dulce). I. II. Boletín de la Sociedad Española de Historia Natural 29: 251-254; 361-364.

González Guerrero, P. 1930. Dos Cianofíceas de agua dulce de Cataluña. Cavanillesia 3: 55-56.

González Guerrero, P. 1930. Datos sobre la ficoflora de agua dulce hispano-marroquí. Boletín de la Sociedad Española de Historia Natural 30: 411-416.

González Guerrero, P. 1930. Algas del río Zújar (Badajoz). Boletín de la Sociedad Española de Historia Natural 30: 223-227.

González Guerrero, P. 1931. Algunos datos algológicos de la Península Ibérica, de Baleares y de Marruecos (aguadulce). Boletín de la Sociedad Española de Historia Natural 31: 633-639.

González Guerrero, P. 1940. Algas de Ifni (agua dulce). Anales del Jardín Botánico de Madrid 1: 99-105.

González Guerrero, P. 1940. Novedades biológicas en algas de Cuenca. Anales del Jardín Botánico de Madrid 1: 107-140.

González Guerrero, P. 1940. Algas de la República Argentina (agua dulce). Anales del Jardín Botánico de Madrid 1: 141-171.

González Guerrero, P. 1944. Algas de Valencia (1.a serie). Anales del Jardín Botánico de Madrid 5: 304-321.

González Guerrero, P. 1944. La ramificación en Homoeothrix caespitosa (Rab.) Kirchn., var. nov. Argentinensis (Cianofíceas) de la República Argentina. Anales del Jardín Botánico de Madrid 5: 322-332.

González Guerrero, P. 1945. Cianofíceas y algas continentales de Cádiz. Anales del Jardín Botánico de Madrid 6: 237-335.

González Guerrero, P. 1946. Algas invernales gaditanas. Anales del Jardín Botánico de Madrid 7: 433-456.

González Guerrero, P. 1947. Nuevos datos ficológicos de España. Anales del Jardín Botánico de Madrid 8: 265-283.

González Guerrero, P. 1949. Observaciones ficobiológicas en la Sierra do Géres (Portugal). Portugaliae acta biologica, série B: sistemática, ecologia, biogeografia e paleontologia, vol. J. Henriques: 17-136.

González Guerrero, P. 1950. Algas y Cianofíceas de la Sierra do Géres, I. Agronomia Lusitana 12: 15-79. 
González Guerrero, P. 1950. Hallazgos ficológicos en nuestra costa. Anales del Instituto Botánico A.J. Cavanilles 10: 191-230.

González Guerrero, P. 1964. Nuevas especies de agua dulce italoespañolas. Giornale botanico italiano 71: 527-543. https://doi. org/10.1080/11263506409440470.

González Guerrero, P. 1964. Algas de la isla de los Osos (Noruega). Anales del Instituto Botánico A.J. Cavanilles 22: 159-184, 2 pl.

González Guerrero, P. 1965. Algas de la sílice (Guadarrama). Anales del Instituto Botánico A.J. Cavanilles 23: 107-43.

Guiry, M.D. \& Guiry, G.M. 2016. AlgaeBase. World-wide electronic publication. National University of Ireland, Galway.

Lawrence, G.H.M., Buchheim, A.F.G., Daniels, G.S. \& Dolezal, H. (eds.). 1968. Botanico-Periodicum-Huntianum. Hunt Institute for Botanical Documentation, Pittsburgh.

Pando, F. \& al. [2004.] BIBMASTER (3.7): A database application for biodiversity projects: taxa-level information, nomenclature, literature and specimen management. [http://www.gbif.es/bibmaster/bibmaster.php.]

Stafleu, F.A. \& Cowan, R.S. 1976. Taxonomic literature ed. 2. Volume I: A-G. Regnum vegetabile 94.

Stafleu, F.A. \& Cowan, R.S. 1979. Taxonomic literature, ed. 2. Volume II: H-Le. Regnum vegetabile 98.
Stafleu, F.A. \& Cowan, R.S. 1981. Taxonomic literature ed. 2. Volume III: Lh-O. Regnum vegetabile 105.

Stafleu, F.A. \& Cowan, R.S. 1983. Taxonomic literature ed. 2. Volume IV: P-Sak. Regnum vegetabile 110.

Stafleu, F.A. \& Cowan, R.S. 1985. Taxonomic literature ed. 2. Volume V: Sal-Ste. Regnum vegetabile 112.

Stafleu, F.A. \& Cowan, R.S. 1985. Taxonomic literature ed. 2. Volume VI: Sti-Vuy. Regnum vegetabile 115.

Stafleu, F.A. \& Cowan, R.S. 1985. Taxonomic literature ed. 2. Volume VII: W-Z. Regnum vegetabile 116.

Stafleu, F.A. \& Menennga, E.A. 1992. Taxonomic literature ed. 2. Supplement I: A-Ba. Regnum vegetabile 125.

Stafleu, F.A. \& Menennga, E.A. 1993. Taxonomic literature ed. 2. Supplement II: Be-Bo. Regnum vegetabile 130.

Stafleu, F.A. \& Menennga, E.A. 1995. Taxonomic literature ed. 2. Supplement III: Br-Ca. Regnum vegetabile 132.

Stafleu, F.A. \& Menennga, E.A. 1997. Taxonomic literature ed. 2. Supplement IV: Ce-Cz. Regnum vegetabile 134.

Stafleu, F.A. \& Menennga, E.A. 1998. Taxonomic literature ed. 2. Supplement V: Da-Di. Regnum vegetabile 135.

Valenzuela, S. \& Pérez-Cirera, J.L. 1982. El herbario de algas marinas españolas de F. Miranda. Collectanea Botanica 13: 945-975. 